\title{
Pseudogap-Driven Sign Reversal of the Hall Effect
}

\author{
D. V. Evtushinsky, ${ }^{1}$ A. A. Kordyuk, ${ }^{1,2}$ V. B. Zabolotnyy, ${ }^{1}$ D. S. Inosov, ${ }^{1}$ B. Büchner, ${ }^{1}$ H. Berger, ${ }^{3}$ \\ L. Patthey, ${ }^{4}$ R. Follath, ${ }^{5}$ and S. V. Borisenko ${ }^{1}$ \\ ${ }^{1}$ Institute for Solid State Research, IFW Dresden, P.O. Box 270116, D-01171 Dresden, Germany \\ ${ }^{2}$ Institute of Metal Physics of National Academy of Sciences of Ukraine, 03142 Kyiv, Ukraine \\ ${ }^{3}$ Institut de Physique Appliquée, Ecole Politechnique Féderale de Lausanne, CH-1015 Lausanne, Switzerland \\ ${ }^{4}$ Swiss Light Source, Paul Scherrer Institut, CH-5234 Villigen, Switzerland \\ ${ }^{5}$ BESSY GmbH, Albert-Einstein-Strasse 15, 12489 Berlin, Germany
}

(Received 13 March 2008; published 11 June 2008)

\begin{abstract}
We present a calculation of the Hall coefficient in $2 \mathrm{H}-\mathrm{TaSe}_{2}$ and $2 \mathrm{H}-\mathrm{Cu}_{0.2} \mathrm{NbS}_{2}$ based on their electronic structure extracted from angle-resolved photoemission spectra. The well-known semiclassical approach, based on the solution of the Boltzmann equation, yields the correct value for the normal-state Hall coefficient. Entering the charge density wave state results in the opening of the pseudogap and redistribution of the spectral weight. Accounting for this allows us to reproduce the temperature dependence of the Hall coefficient, including the prominent sign change, with no adjustable parameters.
\end{abstract}

DOI: 10.1103/PhysRevLett.100.236402

While in simple metals the Hall coefficient is essentially temperature independent, in unconventional materials it can exhibit strong variations with temperature. In particular, in the transition metal dichalcogenides, known as charge density wave (CDW) bearing compounds, the Hall coefficient changes sign from positive to negative soon after the transition into a CDW state [1,2]. A similar sign change of the Hall coefficient has recently been discovered in high temperature superconductors (HTSC) [3]. Another common departure of the CDW and HTSC compounds from conventional solid state theory is the presence of a pseudogap in their excitation spectra [4-7]. The pseudogap results in a depletion of electronic states at the Fermi level. Since the charge dynamics in the crystal is restricted to a narrow energy range around the Fermi level, the opening of the pseudogap reduces the effective number of charge carriers that apparently affects transport properties of the solid. Since the pseudogap can be highly anisotropic [6,7], the quantitative investigation of this effect requires a momentum-resolved experimental technique. In this Letter we suggest a procedure to calculate the Hall coefficient from angle-resolved photoemission spectroscopy (ARPES) data [8] and show that the origin of its sign change in $2 \mathrm{H}-\mathrm{TaSe}_{2}$ is intimately related to the pseudogap phenomenon.

In our calculations we assume the electric field $\mathbf{E}$ to be parallel to $a b$ plane, and the magnetic field $\mathbf{B}$ to be parallel to the $c$ axis (thus, current $\mathbf{j}$ flows in the $a b$ plane). This experimental geometry is common for investigations of two-dimensional compounds, in particular, the dichalcogenides $[1,2,9,10]$. In the low-field limit, the current density is related to the electrical field by means of the conductivity tensor:

$$
\mathbf{j}=\sigma \mathbf{E}, \quad \sigma=\left(\begin{array}{cc}
\sigma_{x x} & \sigma_{x y} \\
-\sigma_{x y} & \sigma_{x x}
\end{array}\right) .
$$

PACS numbers: 71.45.Lr, 74.25.Fy, 74.25.Jb, 79.60.-i

The components of the conductivity tensor are derived from the solution of the semiclassical Boltzmann equation. Neglecting $k_{z}$ dispersion and taking into account the identity of the $a$ and $b$ axes, $\sigma_{x x}$ and $\sigma_{x y}$ are expressed through the integrals over the Fermi surface in the first Brillouin zone (formulas are given in SI units):

$$
\begin{gathered}
\sigma_{x x}=\frac{e^{2}}{2 \pi L_{c} h} \int \tau(\mathbf{k}) v_{F}(\mathbf{k}) d k \\
\sigma_{x y}=\frac{e^{3} B}{L_{c} h^{2}} \int \frac{\tau^{2}(\mathbf{k}) v_{F}^{2}(\mathbf{k})}{\rho(\mathbf{k})} d k,
\end{gathered}
$$

where $\tau$ is the quasiparticle lifetime, $v_{F}$ is the renormalized Fermi velocity, $\rho$ is the Fermi surface curvature radius, $d k$ is the element of the Fermi surface length, $L_{c}$ is the size of the elementary cell along the $c$ axis, $h$ is the Plank's constant, and $e$ is the elementary charge. Mathematically equivalent formulae, but less convenient for our discussion, can be found in the literature [11-13]. By definition, the Hall coefficient is equal to the Hall electrical field over the magnetic field and the current density: $R_{H} \equiv E_{H} /(B \cdot j)$. In terms of the conductivity tensor it is expressed in the following way:

$$
R_{H}=\frac{\sigma_{x y}}{B \sigma_{x x}^{2}}
$$

ARPES gives us a complete knowledge about the band structure. Therefore the only thing missing to calculate the conductivity tensor from Eqs. (2) and (3) is the transport lifetime $\tau$, which should not be mixed with the quantum lifetime $\tau_{q}$, seen in ARPES [14,15]. If we assume that $\tau$ is momentum independent, then it cancels out and the expression for $R_{H}$ reduces to 


$$
R_{H}=\frac{4 \pi^{2} L_{c}}{e} \frac{\int v_{F}^{2}(\mathbf{k}) / \rho(\mathbf{k}) d k}{\left(\int v_{F}(\mathbf{k}) d k\right)^{2}}
$$

where $R_{H}$ is expressed in $\mathrm{m}^{3} / \mathrm{C}$ [16]. For $2 H-\mathrm{TaSe}_{2}, L_{c}=$ $12.7 \AA$ [17], and all other quantities that enter Eq. (5) can be extracted straightforwardly from ARPES spectra as $v_{F}$ is seen in the energy-momentum cuts [15,18], $\rho$ and the integration path are seen in the Fermi surface maps [19].

It is well known that for conventional metals Eq. (5) yields a result consistent with direct measurements of $R_{H}$ [20]. We find that it also provides good agreement with the experiment for $\mathrm{NbS}_{2}$. In case of $\mathrm{TaSe}_{2}$ Eq. (5) provides the correct $R_{H}$ at high temperatures, but becomes inapplicable at lower temperatures, at the Fermi surface reconstruction onset. Formula (2) and (3), and, hence, (5) imply that all energy bands are equally and uniformly populated with electrons. Although this assumption often holds, a complex picture of the spectral weight distribution does not appear to be a rare occasion for unconventional materials $[4,7,22]$. For such a case, Eq. (5) can be modified by introducing a factor $D(\mathbf{k})$ that takes into account the distribution of the spectral weight, i.e., the behavior of the density of states (DOS) near the Fermi level:

$$
R_{H}=\frac{4 \pi^{2} L_{c}}{e} \frac{\int D(\mathbf{k}) v_{F}^{2}(\mathbf{k}) / \rho(\mathbf{k}) d k}{\left(\int D(\mathbf{k}) v_{F}(\mathbf{k}) d k\right)^{2}}
$$

where

$$
D(\mathbf{k})=\int_{-\infty}^{+\infty} \operatorname{DOS}_{\mathbf{k}}(\omega)\left(-\frac{\partial f(\omega)}{\partial \omega}\right) d \omega,
$$

which is the temperature weighted DOS at the Fermi level, $f(\omega)$ is the Fermi function, and $\omega$ is the binding energy. a)

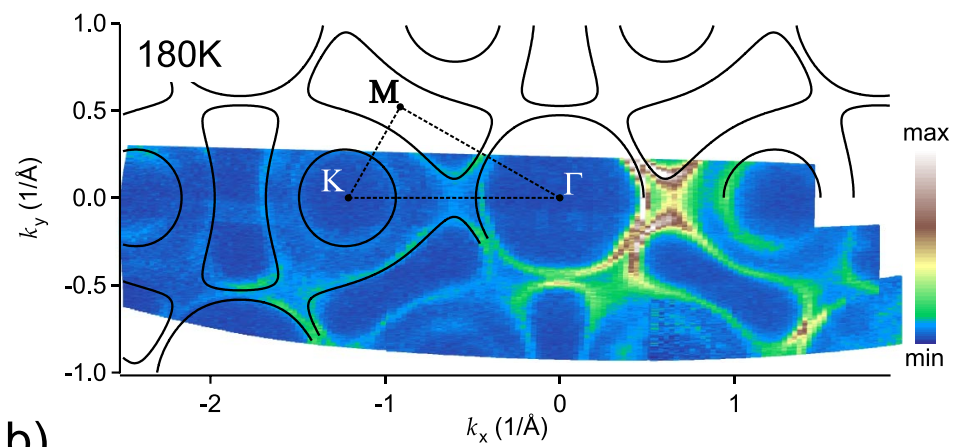

b)

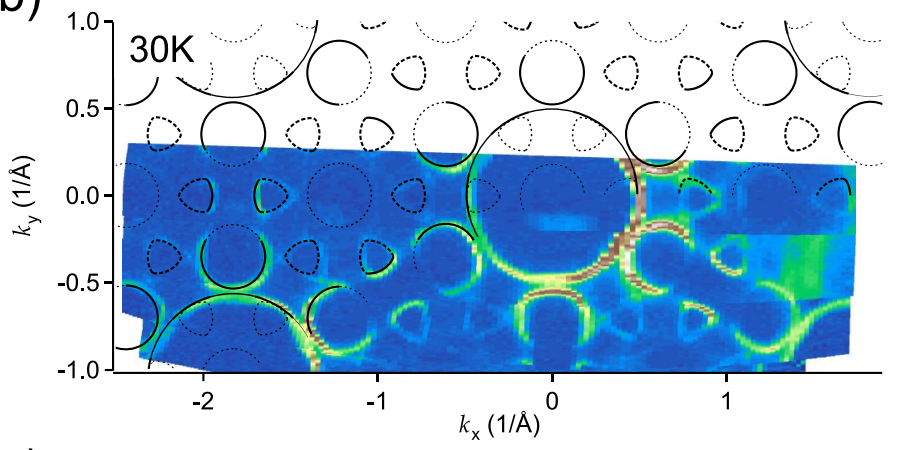

e)

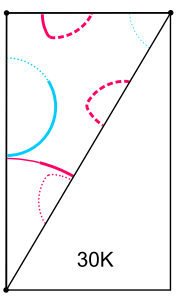

$\mathrm{TaSe}_{2}$

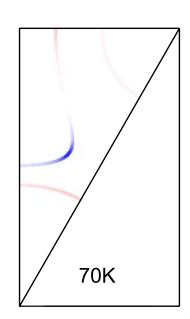

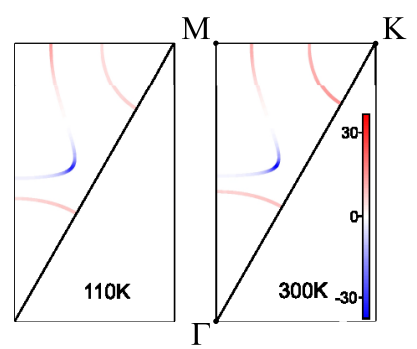

$\mathrm{Cu}_{0.2} \mathrm{NbS}_{2}$

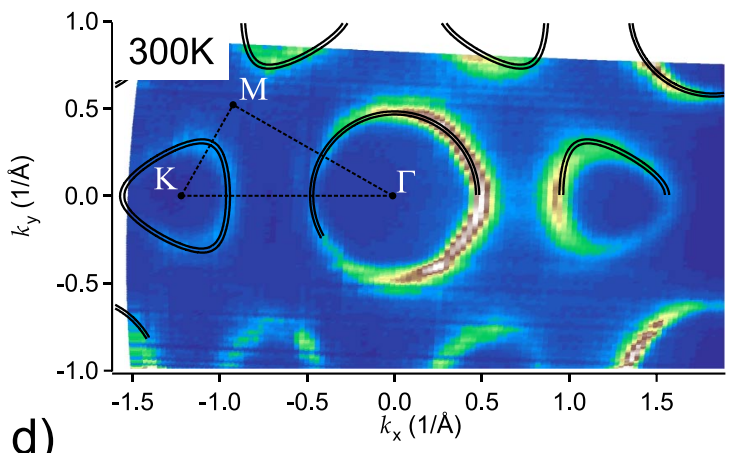

d)

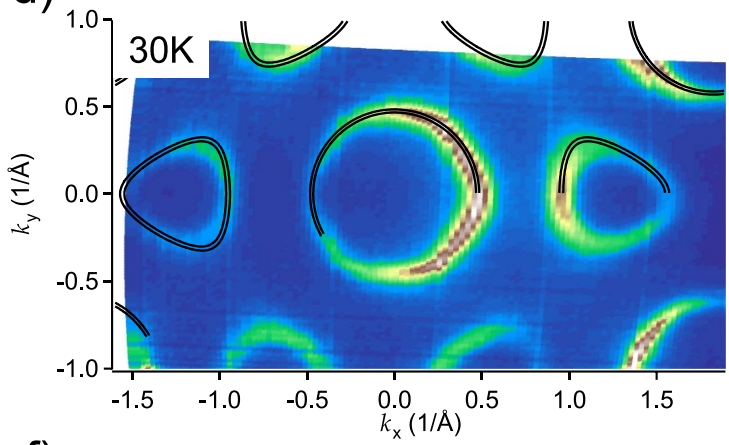

f)

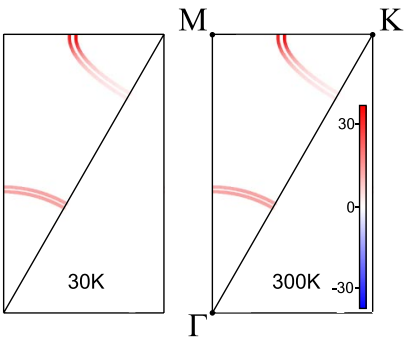

FIG. 1 (color online). Evolution of the Fermi surface of $\mathrm{TaSe}_{2}$ and $\mathrm{Cu}_{0.2} \mathrm{NbS}_{2}$ with temperature. The Fermi surface of TaSe $e_{2}$ changes topology with cooling (a, b). Absence of changes and uniform spectral weight distribution in the spectra of $\mathrm{Cu}_{0.2} \mathrm{NbS}_{2}(\mathrm{c}),(\mathrm{d}) . \mathrm{Solid}$ black lines in (a),(c),(d) are the tight-binding fit to the data. For the CDW-reconstructed Fermi surface (b) different types of guidelines represent the spectral weight distribution: the stronger line corresponds to the higher spectral weight. The relative contribution to $\sigma_{x y}$ from different parts of the Fermi surface at different temperatures is shown in the irreducible part of the Brillouin zone for TaSe $\mathrm{B}_{2}$ (e) and $\mathrm{Cu}_{0.2} \mathrm{NbS}_{2}$ (f). 


\section{Directly measured}

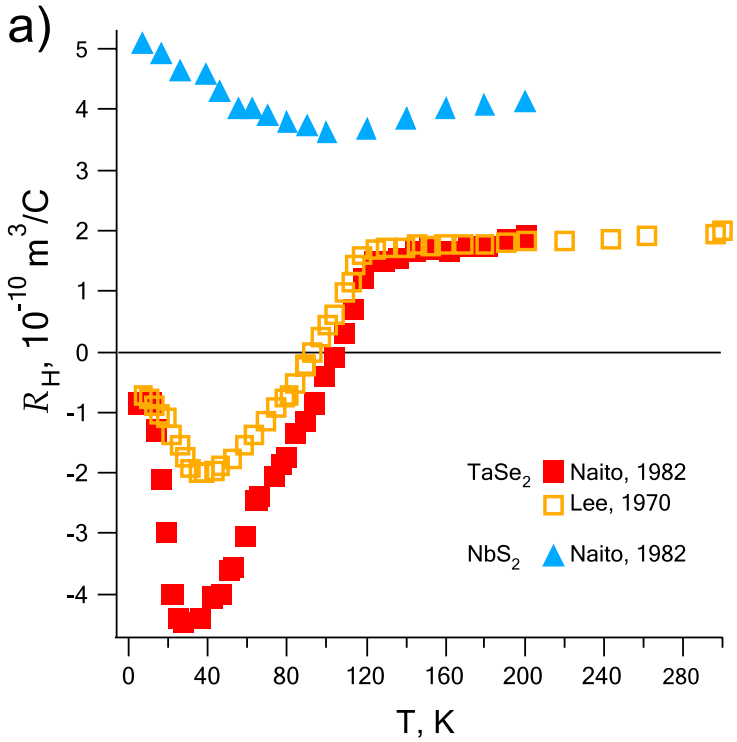

Calculated from ARPES

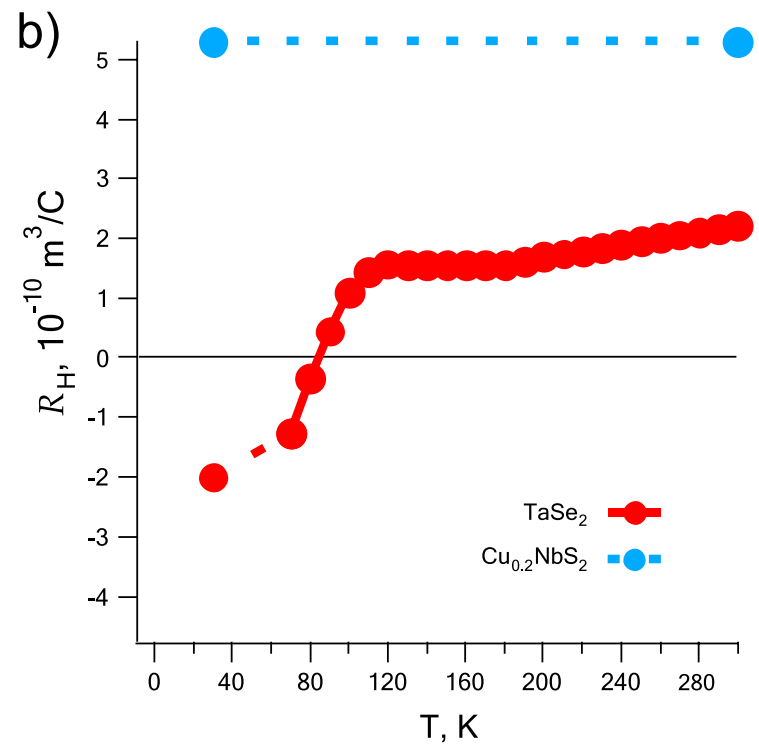

FIG. 2 (color online). The temperature dependence of the Hall coefficient. The Hall coefficient in $\mathrm{NbS}_{2}$ has a weak temperature dependence, while in $\mathrm{TaSe}_{2}$ it changes sign (a). The Hall coefficient of $\mathrm{Cu}_{0.2} \mathrm{NbS}_{2}$, calculated in approximation of the equally "populated" bands, agrees well with the directly measured one [23], while in the case of TaSe $\mathrm{C}_{2}$ one should take into account the spectral weight redistribution and the opening of the pseudogap (b). The discrepancy between two experimental curves for TaSe ${ }_{2}$ is due to the charge density wave suppression by impurities.

Note that in the simplest case $\operatorname{DOS}_{\mathbf{k}}(\omega)=1$, and $D(\mathbf{k})=$ $f(-\infty)-f(+\infty)=1$, so we arrive back at formula (5). In case of the pseudogap-modified spectra, based on the experimental data, we modeled the DOS using the following function:

$$
\operatorname{DOS}_{\mathbf{k}}(\omega)= \begin{cases}1, & |\omega| \geq 2 \Delta \\ |\omega| / 2 \Delta, & |\omega|<2 \Delta\end{cases}
$$

To obtain the experimental input on the electronic structure of $\mathrm{TaSe}_{2}$ and $\mathrm{Cu}_{0.2} \mathrm{NbS}_{2}$ we carried out a series of ARPES measurements for temperatures ranging from 300 down to $30 \mathrm{~K}$ [see Figs. 1(a) and 1(b) and, for further details, Ref. [7]]. $\mathrm{TaSe}_{2}$ undergoes a transition to the incommensurate CDW state at $122 \mathrm{~K}$ and to the commensurate one at $90 \mathrm{~K}$. In the spectra of $\mathrm{TaSe}_{2}$ the pseudogap is already present at room temperature, and begins to increase sharply upon the transition into the incommensurate CDW state, evolving to the band gap in the commensurate CDW state [7]. The magnitude of the pseudogap depends on the position in the Brillouin zone. In the case of $\mathrm{TaSe}_{2}$ the $K$ barrel is affected by the pseudogap most of all, so its contribution to the Hall coefficient has the strongest variation with temperature, and is the primary reason for the Hall coefficient to change sign. Fermi surface reconstruction also implies opening of the pseudogap on the parts of the bone-shaped sheet around the $M$ point and a fading of the $\Gamma$ barrel $\left(\operatorname{DOS}_{\mathbf{k}}(\omega)=\right.$ const $\left.<1\right)$ near the point where it approaches the $M$ bone; see Figs. 1(a) and 1(b) and Ref. [7]. In Fig. 1(e) the contribution to $\sigma_{x y}$ from different parts of the Fermi surface is shown for several temperatures. The above described procedure was also used in conjunction with $\mathrm{Cu}_{0.2} \mathrm{NbS}_{2}$ that exhibits no CDW. As follows from the spectra, the electronic structure exhibits no considerable temperature dependence [Figs. 1(c), 1(d), and 1(f)], and is characterized by a uniform distribution of the spectral weight. The calculated Hall coefficient of $\mathrm{Cu}_{0.2} \mathrm{NbS}_{2}$ [23] shows a weak temperature dependence. Comparing the result of the calculations with the experimental measurements we find good agreement for both the studied compounds [Fig. 2], which implies the implementation of the pseudogap effect into the semiclassical formula is correct.

In conclusion, we have shown that the suppression of the spectral weight at the Fermi level and its nonuniform distribution over the Fermi surface contours, which are related to the pseudogap formation and consequent Fermi surface folding upon entering the CDW state, are indispensable for attaining a quantitative understanding of the temperature dependence of the Hall coefficient in $\mathrm{TaSe}_{2}$. Our findings hint that accounting for the pseudogap and the Fermi surface reconstruction phenomenon may also be fruitful for understanding other physical properties of CDW systems and unconventional superconductors [3,24].

We thank R. Hübel for technical support and $M$. Rümmeli for careful reading of the manuscript. The project is part of the FOR538 and was supported by the DFG under Grants No. KN393/4 and No. BO1912/2-1. ARPES experiments were performed using the " 13 ARPES" end station at the Berliner Elektronenspeicherring-Gesellschaft für 
Synchrotron Strahlung m.b.H. (BESSY) and at the Swiss Light Source (SLS).

[1] H. N. S. Lee et al., J. Solid State Chem. 1, 190 (1970).

[2] M. Naito and S. Tanaka, J. Phys. Soc. Jpn. 51, 219 (1982).

[3] D. LeBoeuf et al., Nature (London) 450, 533 (2007).

[4] A. Kordyuk et al., arXiv:0801.2546 [Phys. Rev. Lett. (to be published)].

[5] T. Timusk et al., Rep. Prog. Phys. 62, 61 (1999).

[6] H. Ding et al., Nature (London) 382, 51 (1996).

[7] S. V. Borisenko et al., Phys. Rev. Lett. 100, 196402 (2008); D. S. Inosov et al., arXiv:0805.4105 [New J. Phys. (to be published)].

[8] Until recently, band calculations have failed to precisely reproduce the Fermi surface geometry of $\mathrm{TaSe}_{2}$. Moreover, the evolution of the electronic structure from the normal to CDW state still remains an open issue. For these reasons the transport properties cannot be calculated a priori.

[9] R. A. Craven and S.F. Meyer, Phys. Rev. B 16, 4583 (1977).

[10] V. Vescoli, L. Degiorgi, H. Berger, and L. Forro, Phys. Rev. Lett. 81, 453 (1998).

[11] T. P. Beaulac, F. J. Pinski, and P. B. Allen, Phys. Rev. B 23, 3617 (1981).

[12] W. H. Butler, Phys. Rev. B 29, 4224 (1984).

[13] N. P. Ong, Phys. Rev. B 43, 193 (1991).

[14] P. T. Coleridge, Phys. Rev. B 44, 3793 (1991).

[15] D. V. Evtushinsky et al., Phys. Rev. B 74, 172509 (2006).

[16] Note that in the simplest case of electronlike round Fermi surface Eqs. (2)-(5) yield well-known Drude formulas:
$R_{H}=-1 / n e, \sigma_{x x}=n e^{2} \tau / m$ where $n=\left(2 \pi^{2} L_{c}\right)^{-1} \times$ $\int f(\epsilon(\mathbf{k})) d^{2} \mathbf{k}$ and $m=\hbar k_{F} / v_{F}$.

[17] D. E. Moncton et al., Phys. Rev. B 16, 801 (1977).

[18] A. Kordyuk et al., Phys. Rev. B 71, 214513 (2005); V. B. Zabolotnyy et al., Phys. Rev. Lett. 96, 037003 (2006).

[19] S. V. Borisenko et al., Phys. Rev. B 64, 094513 (2001).

[20] In the case of spherically symmetric electronic structure, that is a perfect approximation for potassium, sodium and rubidium, one arrives at a simple formula for the Hall coefficient: $R_{H}=-3 \pi^{2} e^{-1} k_{F}^{-3}, k_{F}$ values for alkali metals can be found in the literature [21]. Also in the literature one can find calculations for metals with more complicated Fermi surfaces, e.g., copper and niobium [11]. The calculated Hall coefficient agrees with the experimental one within about $10 \%$, as shown in the following table:

\begin{tabular}{cll}
$R_{H}\left(10^{-10} \mathrm{~m}^{3} / \mathrm{C}\right)$ & Calculation & Experiment \\
\hline $\mathrm{Na}$ & -2.38 & -2.50 \\
$\mathrm{~K}$ & -4.49 & -4.20 \\
$\mathrm{Rb}$ & -5.4 & -5.0 \\
$\mathrm{Cu}$ & -0.530 & -0.517 \\
$\mathrm{Nb}$ & +0.752 & +0.875
\end{tabular}

[21] A. vom Felde, J. Sprosser-Prou, and J. Fink, Phys. Rev. B 40, 10181 (1989); T. Schneider et al., Phys. Kondens. Mater. 6, 135 (1967).

[22] C. Kusko, R. S. Markiewicz, M. Lindroos, and A. Bansil, Phys. Rev. B 66, 140513(R) (2002).

[23] For pure $\mathrm{NbS}_{2}$ we expect Hall coefficient to be roughly $20 \%$ less than for $\mathrm{Cu}_{0.2} \mathrm{NbS}_{2}$.

[24] S. Gnanarajan and R.F. Frindt, Phys. Rev. B 33, 1443 (1986). 